International Journal of Pure and Applied Mathematics

Volume 105 No. 2 2015, 213-221

ISSN: 1311-8080 (printed version); ISSN: 1314-3395 (on-line version)

url: http://www.ijpam.eu

doi: http://dx.doi.org/10.12732/ijpam.v105i2.8

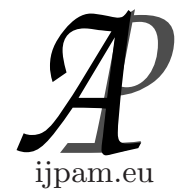

\title{
STABILITY ANALYSIS OF THE EULER DISCRETIZATION FOR THE HARVESTING LESLIE-GOWER PREDATOR-PREY MODEL
}

\author{
Prisca Danik Ernawati ${ }^{1}$, Isnani Darti ${ }^{2}$ \\ ${ }^{1,2}$ Department of Mathematics \\ Brawijaya University \\ Jl. Veteran Malang, 65145, INDONESIA
}

\begin{abstract}
In this paper, a harvesting Leslie-Gower equations is discretized using the forward Euler method. The dynamical properties of the resulted discrete system is then analysed. It is shown that the discrete system has exactly the same equilibria as those of original continuous equation. However, the stability of positive equilibrium is dynamically consistent with its continuous version only for relatively small time step. Such behaviour is confirmed by our numerical simulations. Furthermore, our numerical simulations show when the time step does not satisfy the stability condition, the discrete system has more complicated dynamics such as period- $2^{n}$-orbits and chaos.
\end{abstract}

AMS Subject Classification: 37N25, 92D40

Key Words: difference equation, forward Euler method, dynamically consistent, asymptotically stable

\section{Introduction}

Recently, Zhang et al. [1] introduced the following Leslie-Gower predator-prey

Received: September 1, 2015

(c) 2015 Academic Publications, Ltd. url: www.acadpubl.eu 
model with harvesting

$$
\begin{gathered}
\frac{d H}{d t}=\left(r_{1}-a_{1} P-b_{1} H\right) H-c_{1} H, \\
\frac{d P}{d t}=\left(r 2-a_{2} \frac{P}{H}\right) P-c_{2} P,
\end{gathered}
$$

where $H$ and $P$ denote the density of prey and predator, respectively. The growth of prey obeys the logistic law with intrinsic growth rate $r_{1}$ and carrying capacity $r_{1} / b_{1}$. The predator is also assumed to grow logistically with intrinsic growth rate $r_{2}$ but its carrying capacity depends on the number of prey. Here $a_{1}$ and $a_{2}$ are the predation rate and the measure of the food quantity that the prey provides converted to predator birth. To ensure the sustainable development, it is assumed that the harvesting coefficients should satisfy $0<c_{i}<r_{i}, i=1,2$. Due to biological nature and to avoid the singularity, the solutions of system (1) has to satisfy the positivity condition that $H(t)>0$ and $P(t) \geq 0$ for all $t \geq 0$.

Zhang et al. [1] showed that system (1) admits a unique positive equilibrium $E^{*}\left(H^{*}, P^{*}\right)$ where

$$
H^{*}=\frac{\left(r_{1}-c_{1}\right) a_{2}}{a_{1}\left(r_{2}-c_{2}\right)+a_{2} b_{1}} ; P^{*}=\frac{\left(r_{1}-c_{1}\right)\left(r_{2}-c_{2}\right)}{a_{1}\left(r_{2}-c_{2}\right)+a_{2} b_{1}} .
$$

The positive equilibrium $E^{*}\left(H^{*}, P^{*}\right)$ obviously satisfies the equalities

$$
r_{1}-c_{1}=a_{1} P^{*}+b_{1} H^{*}, r_{2}-c_{2}=a_{2} \frac{P^{*}}{H^{*}} .
$$

Model (1) also has a boundary equilibrium $E^{0}\left(H^{0}, 0\right)$ where $H^{0}=\left(r_{1}-c_{1}\right) / b_{1}$. Zhang et al. [1] proved that the positive equilibrium $E^{*}\left(H^{*}, P^{*}\right)$ is asymptotically stable while the boundary equilibrium is unstable.

Considering the need of scientific computation and real time simulation, it is often necessary to discretize model (1) in order to obtain its numerical solutions. The are a number of numerical methods for solving a system of first order nonlinear differential equations. Basically the numerical discretizations transform the continuous dynamical system into a discrete dynamical system. Hence, the obtained discrete model should maintain the dynamical properties of the original continuous model. Darti and Suryanto [2] has implemented a nonstandard finite difference (NSFD) method to solve system (1). It is shown that the discrete system derived from the NSFD method preserves some important features of model (1), irrespective of the size of numerical time step. Recently, 
Fayeldi [3] has implemented much simpler numerical method, i.e., the forward Euler method, to discretize model (1) and studied the dynamical behaviours of the resulted discrete model. When applying the forward Euler method, he used a specific numerical time step, i.e., $(h=1)$ and showed that the stability of the positive equilibrium is determined by parameters of model (1). However, it is known that the stability properties of a discrete model obtained by the Euler method are dependent on the numerical time step (see [4], [5], [6], [7], [8], [9], [10]). Hence, in this paper we will apply the forward Euler method with arbitrary time step and establish the sufficient condition such that the Euler discretization of system (1) maintains the stability properties of the original continuous system.

\section{Discrete Model: Equilibria and Their Stability}

Applying the forward Euler scheme to model (1) we obtain the following discretetime Leslie-Gower predator-prey model with harvesting

$$
\begin{gathered}
H_{n+1}=\left(1+h\left(r_{1}-a_{1} P_{n}-b_{1} H_{n}-c_{1}\right)\right) H_{n}, \\
P_{n+1}=\left(1+h\left(r_{2}-a_{2} \frac{P_{n}}{H_{n}}-c_{2}\right)\right) P_{n},
\end{gathered}
$$

where $h$ is the step size.

We firstly discuss the existence of the equilibria of discrete model (2). From system (2), we obviously have the following results.

Lemma 1. The discrete model (2) has two equilibria, namely the boundary equilibrium $E^{0}\left(H^{0}, 0\right)$ and the positive equilibrium $E^{*}\left(H^{*}, P^{*}\right)$ which are independent of $h$.

Hence, the equilibria of discrete model (2) are exactly the same as those of continuous model (1). The stability of these equilibria will be studied locally by using a linearization method and Jacobian matrix. Jacobian matrix of the linearized discrete model (2) at an equilibrium $\widehat{E}(\widehat{H}, \widehat{P})$ is

$$
J(\widehat{E})=\left(\begin{array}{cc}
1+h\left(r_{1}-a_{1} \widehat{P}-2 b_{1} \widehat{H}-c_{1}\right) & -h a_{1} \widehat{H} \\
h a_{2}\left(\frac{\widehat{P}}{\widehat{H}}\right)^{2} & 1+h\left(r_{2}-2 a_{2} \frac{\widehat{P}}{\widehat{H}}-c_{2}\right)
\end{array}\right) .
$$

The equilibrium $(\widehat{H}, \widehat{P})$ is asymptotically stable if all eigenvalues $\left(\lambda_{i}, i=1,2\right)$ of the associated Jacobian matrix (3) satisfy $\left|\lambda_{i}\right|<1$. 
If the boundary equilibrium $E^{0}\left(H^{0}, 0\right)$ is substituted into the Jacobian matrix (3), then it can be check easily that its eigenvalues are $\lambda_{1}=1+h\left(c_{1}-r_{1}\right)$ and $\lambda_{2}=1+h\left(r_{2}-c_{2}\right)$. Since $c_{i}<r_{i}, i=1,2$, then it is obvious that $\lambda_{1}<1$ while $\lambda_{2}>1$. Therefore the boundary equilibrium $E^{0}$ is unstable for any $h$.

The Jacobian matrix of model (2) at the positive equilibrium $E^{*}\left(H^{*}, P^{*}\right)$ is

$$
J\left(E^{*}\right)=\left(\begin{array}{cc}
1-\frac{h a_{2} b_{1}\left(r_{1}-c_{1}\right)}{a_{1}\left(r_{2}-c_{2}\right)+a_{2} b_{1}} & -\frac{h a_{1} a_{2}\left(r_{1}-c_{1}\right)}{a_{1}\left(r_{2}-c_{2}\right)+a_{2} b_{1}} \\
h \frac{\left(r_{2}-c_{2}\right)^{2}}{a_{2}} & 1-h\left(r_{2}-c_{2}\right)
\end{array}\right) .
$$

The corresponding characteristic equation of Jacobian matrix $J\left(E^{*}\right)$ is

$$
F(\lambda)=\lambda^{2}-p \lambda+q
$$

where $p=2-\left(h\left(r_{2}-c_{2}\right)+\frac{a_{2} b_{1}\left(r_{1}-c_{1}\right)}{a_{1}\left(r_{2}-c_{2}\right)+a_{2} b_{1}}\right)$ and $q=q_{2} h^{2}+q_{1} h+1$ with

$$
q_{1}=-\frac{a_{2} b_{1}\left(r_{1}-c_{1}+r_{2}-c_{2}\right)+a_{1}\left(r_{2}-c_{2}\right)^{2}}{a_{1}\left(r_{2}-c_{2}\right)+a_{2} b_{1}}<0
$$

and

$$
q_{2}=\frac{a_{2} b_{1}\left(r_{1}-c_{1}\right)\left(r_{2}-c_{2}\right)}{a_{1}\left(r_{2}-c_{2}\right)+a_{2} b_{1}}+\frac{a_{1}\left(r_{1}-c_{1}\right)\left(r_{2}-c_{2}\right)^{2}}{a_{1}\left(r_{2}-c_{2}\right)+a_{2} b_{1}}>0 .
$$

The stability properties of positive equilibrium $E^{*}$ is determined by the roots of this characteristic equation. To study these characteristic roots, we recall the following lemma [11, 12].

Lemma 2. Roots of the quadratic equation $\lambda^{2}-p \lambda+q=0$ satisfy $\left|\lambda_{i}\right|<1, i=1,2$ if and only if the following three conditions hold:

1. $1+p+q>0$

2. $1-p+q>0$

3. $q<1$

Hence, the positive equilibrium $E^{*}$ is asymptotically stable if and only if Equation (4) satisfies three conditions in Lemma 2. From equation (4), it can be shown that

$$
1-p+q=\frac{a_{2} b_{1} h^{2}\left(r_{1}-c_{1}\right)\left(r_{2}-c_{2}\right)}{a_{1}\left(r_{2}-c_{2}\right)+a_{2} b_{1}}+\frac{a_{1} h^{2}\left(r_{1}-c_{1}\right)\left(r_{2}-c_{2}\right)^{2}}{a_{1}\left(r_{2}-c_{2}\right)+a_{2} b_{1}} .
$$


Since $0<c_{i}<r_{i}, i=1,2$, we always have that $1-p+q>0$. Condition $q<1$ is equivalent to $q_{2} h+q_{1}<0$ which is also equivalent to condition $h<h^{*}$ where

$$
h^{*}=-q_{1} / q_{2}=\frac{a_{2} b_{1}\left(r_{1}-c_{1}+r_{2}-c_{2}\right)+a_{1}\left(r_{2}-c_{2}\right)^{2}}{\left(r_{1}-c_{1}\right)\left(r_{2}-c_{2}\right)\left(a_{2} b_{1}+a_{1}\left(r_{2}-c_{2}\right)\right)} .
$$

Finally, condition $1+p+q>0$ is equivalent to $q_{2} h^{2}+2 q_{1} h+4>0$. By denoting $D=q_{1}^{2}-4 q_{2}$, we consider the following three cases.

Case i: Suppose that $D=0$, then $1+p+q>0$ is satisfied if $h \neq h^{*}$.

Case ii: Suppose that $D<0$, then $1+p+q>0$ is satisfied for any value of $h>0$.

Case iii: Suppose that $D>0$, then $1+p+q>0$ holds if $0<h<h_{1}$ or $h>h_{2}$ where $h_{1}=\frac{-q_{1}-\sqrt{q_{1}^{2}-4 q_{2}}}{q_{2}}>0$ and $h_{2}=\frac{-q_{1}+\sqrt{q_{1}^{2}-4 q_{2}}}{q_{2}}>0$.

It can be checked that $h_{1} \leq h^{*} \leq h_{2}$. Based on the above discussion, we immediately obtain the following theorem.

Theorem 3. The boundary equilibrium $E^{0}$ is always unstable, while the positive equilibrium $E^{*}$ is asymptotically stable if one of the following conditions holds:

1. $q_{1}^{2}-4 q_{2} \leq 0$ and $0<h<h^{*}$

2. $q_{1}^{2}-4 q_{2}>0$ and $0<h<h_{1}$

By comparing the equilibria of discrete model (2) and their stability properties with those of continuous model (1), we conclude that the discrete model is dynamically consistent with the continuous model only for relatively small $h$.

\section{Numerical Simulations}

To illustrate the previous analytical results, we show some results of our numerical simulations. For the simulations, we adopt parameters from Zhang et al. [1], i.e., $r_{1}=1.6, r_{2}=1.0, a_{1}=0.7, a_{2}=1.5, b_{1}=0.3, c_{1}=0.821$ and $c_{2}=0.859$ with initial condition $H(0)=2.0$ and $P(0)=0.5$. Using these parameters we have that $D=0.1688>0$ and therefore, based on the previous analysis, the positive equilibrium $E^{*}(2.1296,0.2002)$ is asymptotically stable only for $h<h_{1} \approx 3.359$. This behaviour can be seen clearly in the bifurcation diagram, see Figure 1. Here we see that $E^{*}$ is stable for $h<h_{1}$. When 

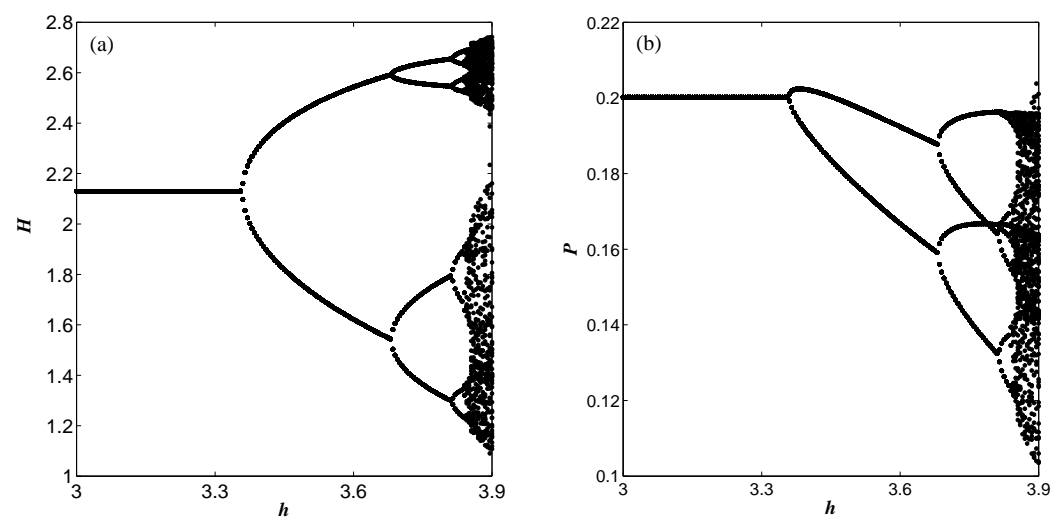

Figure 1: Bifurcation diagram of (a) $H$ and (b) $P$ obtained from system (2) with $r_{1}=1.6, r_{2}=1.0, a_{1}=0.7, a_{2}=1.5, b_{1}=0.3, c_{1}=0.821$, $c_{2}=0.859$ and initial condition $H(0)=2.0$ and $P(0)=0.5$.

$h_{1}<h<3.68$, there is the period-2 orbits. In the range $h \in(3.68,3.835)$ there appear period- 4 orbits and period- 8 orbits. At last, the $2^{n}$ period orbits disappear and the dynamical behaviours are from non-period orbits to the chaotic set with the increasing of $h$. For examples, we plot in Figure 2 and Figure 3 the numerical solutions $H(t)$ and $P(t)$ using $h=3.0,3.5,3.75$ and $h=3.88$ respectively. From these figures we see that $E^{*}$ is a stable equilibrium when $h=3.0$, the solution is convergent to period- 2 orbits when $h=3.5$, the solution is convergent to period- 4 orbits when $h=3.75$ and the solution is non-periodic when $h=3.88$.

\section{Conclusions}

In this paper we discuss the dynamical properties of a discrete Leslie-Gower predator-prey model with harvesting which is derived from the forward Euler method. The discrete model has two equilibria, namely the boundary equilibrium and the positive equilibrium. The boundary equilibrium is always unstable while the stability of positive equilibrium is dependent on the time step $h$. For relatively small time step $h$, the positive equilibrium is asymptotically stable while for higher value of $h$, there appears complex dynamical behaviours such as cascade period-doubling orbits or chaotic orbit. 

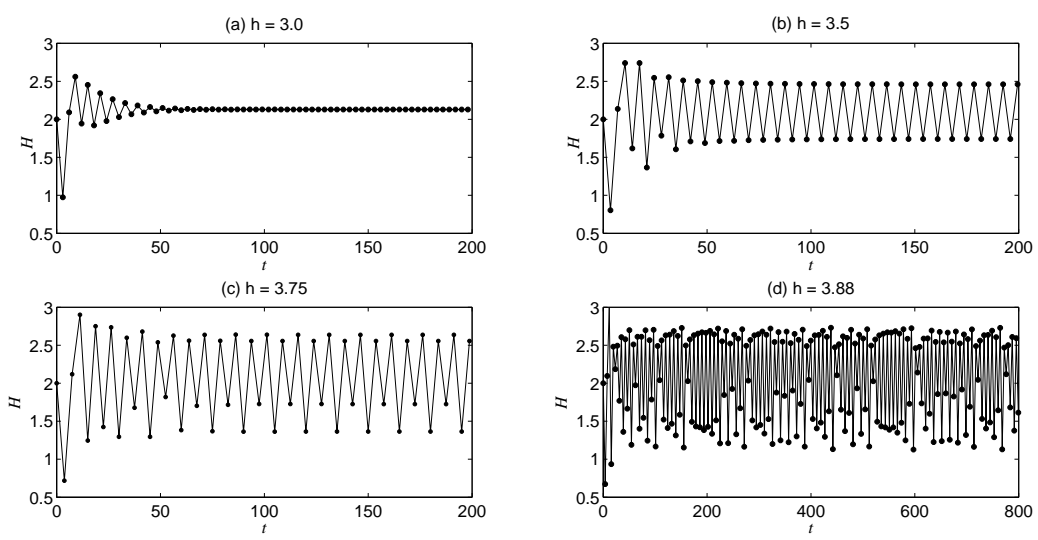

Figure 2: Time series of prey $(H)$ as solutions of system (1) using the forward Euler scheme (2) with $r_{1}=1.6, r_{2}=1.0, a_{1}=0.7, a_{2}=1.5$, $b_{1}=0.3, c_{1}=0.821, c_{2}=0.859$ and initial condition $H(0)=2.0$ and $P(0)=0.5$ using (a) $h=3.0$; (b) $h=3.5$; (c) $h=3.75$ and (d) $h=3.88$.
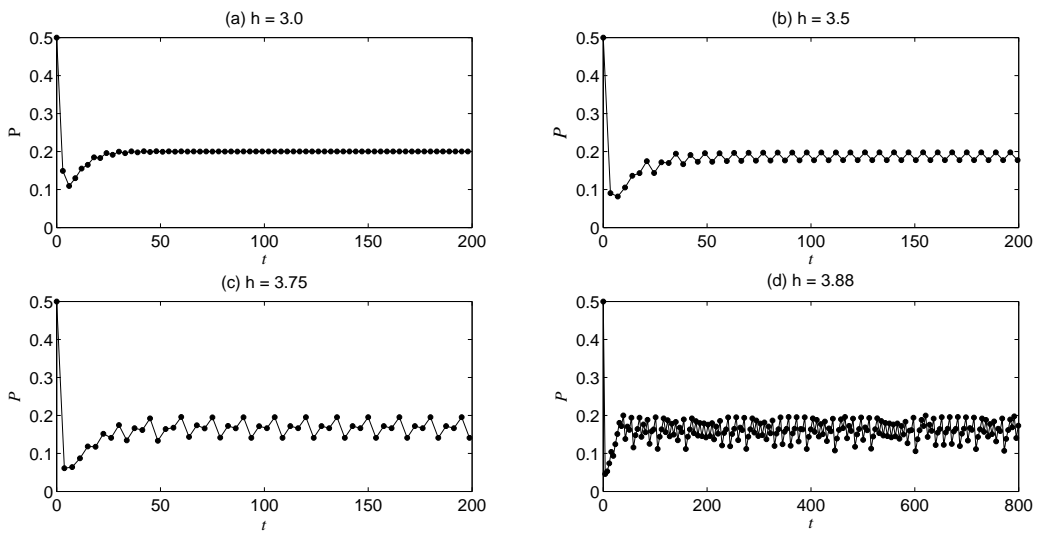

Figure 3: Time series of predator $(H)$ as solutions of system (1) using the forward Euler scheme (2)with $r_{1}=1.6, r_{2}=1.0, a_{1}=0.7, a_{2}=1.5$, $b_{1}=0.3, c_{1}=0.821, c_{2}=0.859$ and initial condition $H(0)=2.0$ and $P(0)=0.5$ using (a) $h=3.0$; (b) $h=3.5$; (c) $h=3.75$ and (d) $h=3.88$. 


\section{Acknowledgments}

The authors would like to express their deepest gratitude to Agus Suryanto, Department of Mathematics, Brawijaya University for the idea, fruitful discussion and suggestion to improve this paper.

\section{References}

[1] N. Zhang, F. Chen, Q. Su, T. Wu, Dynamics behaviors of a Harvesting Leslie-Gower predator-prey model, Discrete Dyn. Nat. Soc., 2011 (2011), Article ID 473949, 14 pages.

[2] I. Darti, A. Suryanto, Stability preserving non-standard finite difference scheme for a harvesting Leslie-Gower predator-prey model, J. Differ. Equ. Appl., 21 No. 6, (2015), 528-534.

[3] T. Fayeldi, Skema numerik persamaan Leslie-Gower dengan pemanenan, Cauchy, 3, No. 4, (2015), 34-38.

[4] T. Fayeldi, A. Suryanto, A. Widodo, Dynamical behaviors of a discrete SIR epidemic model with non-monotone incidence rate, Int. J. Appl. Math. Stat., 47, (2013), 416-23.

[5] Z. Hu, Z. Teng, H. Jiang, Stability analysis in a class of discrete SIRS epidemic models, Nonlinear Anal. Real World Appl., 13, (2012), 20172033.

[6] D. Hu, H. Cao, Bifurcation and chaos in a discrete-time predator-prey system of Holling and Leslie type, Commun. Nonlinear Sci. Numer. Simul., 22 No. 13, (2015), 702-715.

[7] X. Liu, D. Xiao, Complex dynamic behaviors of a discrete-time predatorprey system, Chaos, Solitons $\mathscr{E}$ Fractals, 32 No. 1, (2007), 80-94.

[8] R. Alawia, I. Darti, A. Suryanto, Stability and Bifurcation Analysis of Discrete Partial Dependent Predator-Prey Model with Delay, Appl. Math. Sciences, 7 No. 89, (2013), 4403-4413.

[9] A. Suryanto, Stability analysis of the Euler discretization for SIR epidemic model, AIP Conf. Proc., 1602, (2014), 375-379. 
[10] W. Tan, J. Gao, W. Fan, Bifurcation Analysis and Chaos Control in a Discrete Epidemic System, Discrete Dyn. Nat. Soc., 2015, (2015) Article ID 974868, 13 pages.

[11] F. Brauer, C. Castillo-Chavez, Mathematical models in population biology and epidemiology, Springer, New York (2001).

[12] S. Elaydi, An Introduction to Difference Equations, 3rd ed., Springer, New York (2005). 
